\title{
The Effect on the Gas Selectivity of Gas Sensors by Binder in SWNTs Solution
}

\author{
KIM/SEONGJEEN ${ }^{1}$, GAM/BYUNGMIN ${ }^{1}$, LEE/HOJUNG ${ }^{1}$, HAN/JUNGTAK ${ }^{2}$, LEE/GUNWOONG ${ }^{2}$ \\ ${ }^{1}$ Dept. of Electronic Eng. Kyungnam University \\ 449 Wolyoung-dong, Masan, 631-701, Kyungnam, Korea \\ 2 KERI(Korea Electrotechnology Research Institute) \\ 28 Seongju-dong, Changwon, 641-120, Kyungnam, Korea
}

\begin{abstract}
In this work, we investigated the effect of TEOS and VTMS binders in single walled carbon nanotube(SWNT) solution for imparting selectivity as well as increasing sensitivity to nanotube sensors. Generally binder as organic compound is blended in SWNT solution to adhere to substrate well, and the surface of hydrolyzed binder remain generally functionalized with its own group when it becomes hardened. TEOS and VTMS surrounding SWNTs after hydrolysis remain functionalized to $-\mathrm{OH}$ and $\mathrm{CH}=\mathrm{CH}_{2}$ groups, respectively, Therefore, charge transfer between the carbon nanotubes and adsorbed molecules will be affected, changing their overall electrical conductivity. In this experiment the resistance in the sensors using TEOS decreased for increasing alcohol concentration while it increased in the sensors using VTMS.
\end{abstract}

\section{Introduction}

Since it was experimentally demonstrated that the electrical conductance of carbon nanotubes could be modulated upon exposure of gaseous molecules of $\mathrm{CO}_{2}, \mathrm{NO}_{2}$ and $\mathrm{NH}_{3}$ [1], studies on CNT-based gas sensors have been expanded widely. Today nanotube sensors have shown potential and significant advantages over traditional sensor materials (mainly semiconducting metal oxides) in terms of sensitivity, operation at room temperature, small sizes for device miniaturization and massive sensor arrays.

Lately much emphasis is being placed on developing chemical solution-based deposition technologies of $\mathrm{CNT}$ (carbon nanotube) as an economical alternative to high expensive gas-phase process. In many works [2-3], CNT-based gas sensors have often been fabricated into a thin film configuration rather than into intact single SWNT, multiple SWNTs and CNT array configurations synthesized by arc-discharge, laser ablation and chemical vapor deposition. Solution-based carbon nanotube is easy to be treated relatively and thin films can be formed by various methods such as spin coating, spraying, ink-jetting and imprinting, so it is expected that solution-based CNT gas sensors will be fabricated with easy process for detection of various gases.

In this work, we investigated the effect of binders contained in CNT solution for imparting selectivity as well as increasing sensitivity to nanotube gas sensors. For this, SWNTs/silane sol solutions mixed with different silane binders (TEOS and VTMS in this work) were prepared for depositing SWNTs/silane hybrid 
thin films where the dispersed SWNT network controlled the electrical conductivity of the thin films and where the silane compounds provided various chemical functionalizations on the surface as well as mechanical adhesion. Generally silane compounds as coupling agents are often used in CNT solutions to adhere CNT to substrate well and it has been known that they can be easily functionalized to their own groups on the surface of CNTs after they are hardened by way of the hydrolysis reaction if they are mixed with water. Therefore, it is expected that functionalization by different binders on the surface of CNTs will contribute to the enhancement of gas selectivity.

At present, alcohol gas sensors are fabricated for the use of various purposes such as the detection of VOCs (volatile organic compounds) and the detection of person's breath alcohol applicable to a breath analyzer. Among them, the alcohol gas sensors for the breath alcohol detection have been commercialized broadly at present. Usually commercial alcohol-gas sensors to measure alcohol blood levels should be able to measure samples at low concentrations of the order of a few hundred parts per million. In this work, CNT-based gas sensors were fabricated using SWNTs solution for the breath alcohol detection, and their electrical properties were measured and discussed.

\section{Device fabrication and measurements}

The solution-based CNT gas sensors studied in our experiment were fabricated using tangled bundles of SWNTs. In this work, to form CNT thin film by spray coating, SWNTs(single-walled carbon nanotubes) were dispersed and mixed in diluted ethanol. Since impurities in the form of metallic catalysts, amorphous carbon, fullerenes and graphitic carbon are deposited during the nanotubes synthesis, subsequent steps of purification are required to separate the carbon nanotubes from non-tubular impurities. For acid purification, $100 \mathrm{mg}$ unpurified HiPco SWNTs (CNI, 35\% metal catalyst) were dispersed in $50 \mathrm{ml}$ of $30 \mathrm{vol} \%$ nitric acid and subsequently sonicated for 1 hour and 3hours using water bath sonicator (70W), then the sonicated suspensions were refluxed at boiling condition for 1 hour and 3hours, respectively. The resulting suspensions were diluted and repeatedly washed out in distilled water to eliminate residual acid. Finally, it was filtered through filter paper (Fisher Scientific, No. 1).

We prepared two types of silane sol solutions for comparison by mixing each tetraethoxysilane (TEOS) or vinyltrimethoxysilane (VTMS) in water and ethanol through a sol-gel reaction at $60^{\circ} \mathrm{C}$. The silane sol solutions were then blended with the CNT solution at the concentration of $70 \mathrm{wt} \%$. The fabrication of the CNT/silane hybrid thin films on cover glass substrate was achieved by a spray-coater (Fujimori Co., NVD200 model) with a nozzle size of $1.2 \mathrm{~mm}$ diameter at $70^{\circ} \mathrm{C}$. The hybrid thin films were heated under vacuum for 3 hours (at $150^{\circ} \mathrm{C}$ ) in order to remove the remaining chemicals and cure the silane binder. To form the electrodes of the sensor, Al metallization was followed on the SWNTs thin films with a shadow mask using an evaporator. We measured resistance and time response properties in a probe station. Figure 1 indicates a schematic diagram of the sensor with a real image. 

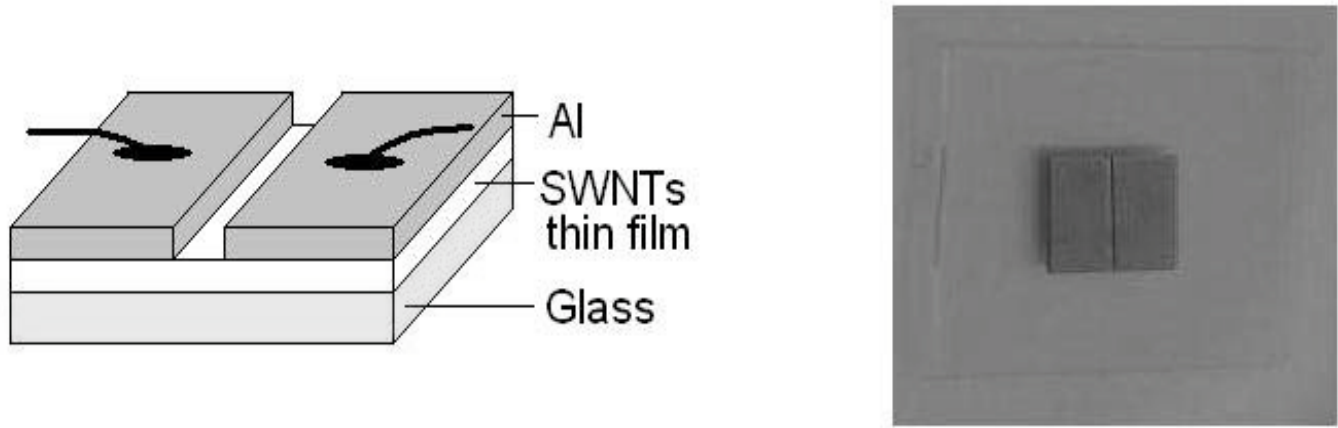

Fig. $1 \mathrm{~A}$ schematic diagram of the sensor with a real image

Electrical conductance was measured with a semiconductor device analyzer and a HP-4280A in a gas chamber. We exposed alcohol vapors evaporated from solutions composed of different ethanol wt $\%$ into the SWCNT layer at $37^{\circ} \mathrm{C}$ equal to the human-body temperature. The sensors were first flushed with clean nitrogen gas before the exposure to vapors, and were measured 30 seconds after exposure to gases. The measurement was repeated for two types of samples.

\section{Results and Discussion}

To see the effect on the selectivity by binder, SWNT-based gas sensors were fabricated with two different SWNT solutions which were blended with TEOS (tetraethylorthosilane) or VTMS (vinyltrimethoxysilane) here, and their resistance response was measured and compared. We formed SWNTs thin film on a cover glass as substrate by spray-coating, followed by hardening and drying.

In this experiment, we examined the response of electrical resistance for alcohol vapor gas. We took ethanol vapor molecules vaporized from ethanol solutions of 0.0 to $0.3 \mathrm{vol} \%$ concentration diluted in pure water where the ethanol vapor molecules were flowed into a chamber with $\mathrm{N}_{2}$ carrier gas in order to keep the situation similar to human breath. The ethanol solutions were kept to $37^{\circ} \mathrm{C}$ close to the temperature of human body. Since ethanol is very volatile and has strong dipole moment, it is expected that the SWNTs will react to these vapors effectively, leading to transfer of considerable electrical charges to SWNTs. We observed the variation in resistance in the sensors using TEOS and VTMS as binder for alcohol concentrations. As shown in Figure 2, a contrary result was found. The resistance in the sensors using TEOS decreased for increasing alcohol concentration while it increased in the sensors using VTMS. 

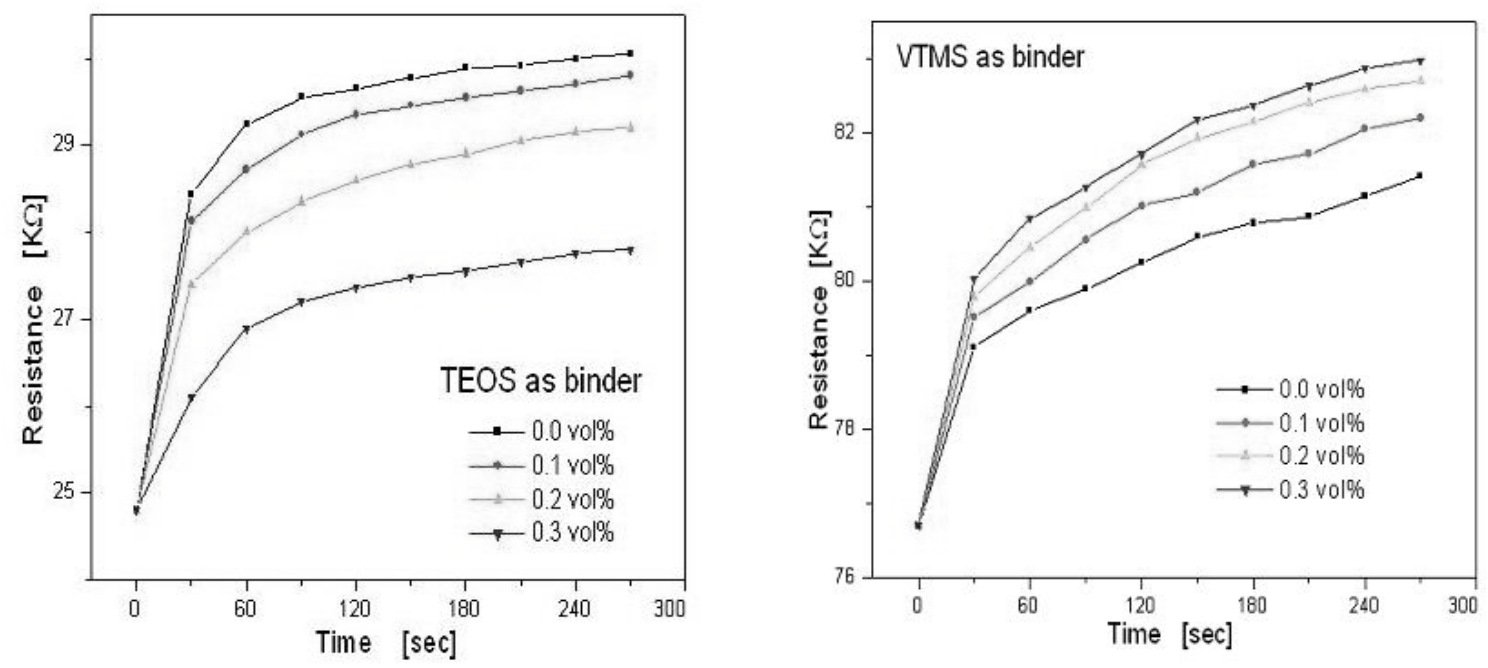

Fig.2 The variation of resistance in the sensors using TEOS and VTMS as binder for alcohol concentrations

The promotion or mediation of charge transfer in CNTs thin film by adsorption of electroactive molecules is a main mechanism in most CNT-based chemical sensors application [4]. If an electron-acceptor type of gas molecules is adsorbed at the surface of SWNTs, the SWNTs thin films will show p-type semiconducting property of increasing conductance while showing decreasing conductance against an electron-donor type of gas molecules. Therefore, the decrease of resistance in the sensor using TEOS was supposed to be related to the charge transfer due to the electron-acceptor characteristic, while the increase of resistance in the sensor using VTMS was supposed to be related to the fact that the surface of the SWNTs was acted like electron-donor. In the result, this indicates that the selectivity can be controlled by varying the type of silane compounds used as binder (e.g., tetraethoxysilane (TEOS), vinyltrimethoxysilane (VTMS)). Moreover, the combination of these silane compounds in sol mixtures will allow the possibility of fabricating highly functional film suitable to gas sensors.

Figure 3 indicates the variations in resistance on the alcohol concentration as a function of the exposure time in the sensor using TEOS and VTMS as binder. At the beginning the sensor using TEOS was responded relatively rapidly and then the variation of the resistance was saturated as the exposure time increased. However, the sensor using VTMS was responded relatively slowly and the variation of the resistance was not saturated during the same duration. In the sensor using TEOS it was observed that the resistance approached up to $90 \%$ of the maximum value after a lapse of $120 \mathrm{sec}$.

\section{Conclusion}

We fabricated alcohol gas sensors composed of SWNTs thin film formed with CNT/silane sol solution, and compared the variation of resistance in the sensors using TEOS and VTMS as binder when they were exposed to alcohol vapor gas. From the two sensors, it was observed that the variation in resistance was opposite. That is, the resistance in the sensor using TEOS for increasing alcohol concentrations 
decreased while the resistance in the sensor using VTMS increased on the contrary. As the result, it was found that the selectivity in a gas sensor could be improved by binder involved in CNT/silane sol solution. In conclusion we suggest that it is possible to fabricate more sensitive and selective functional films for CNT-based gas sensors by controlling binders contained in SWNTs/silane sol solution.
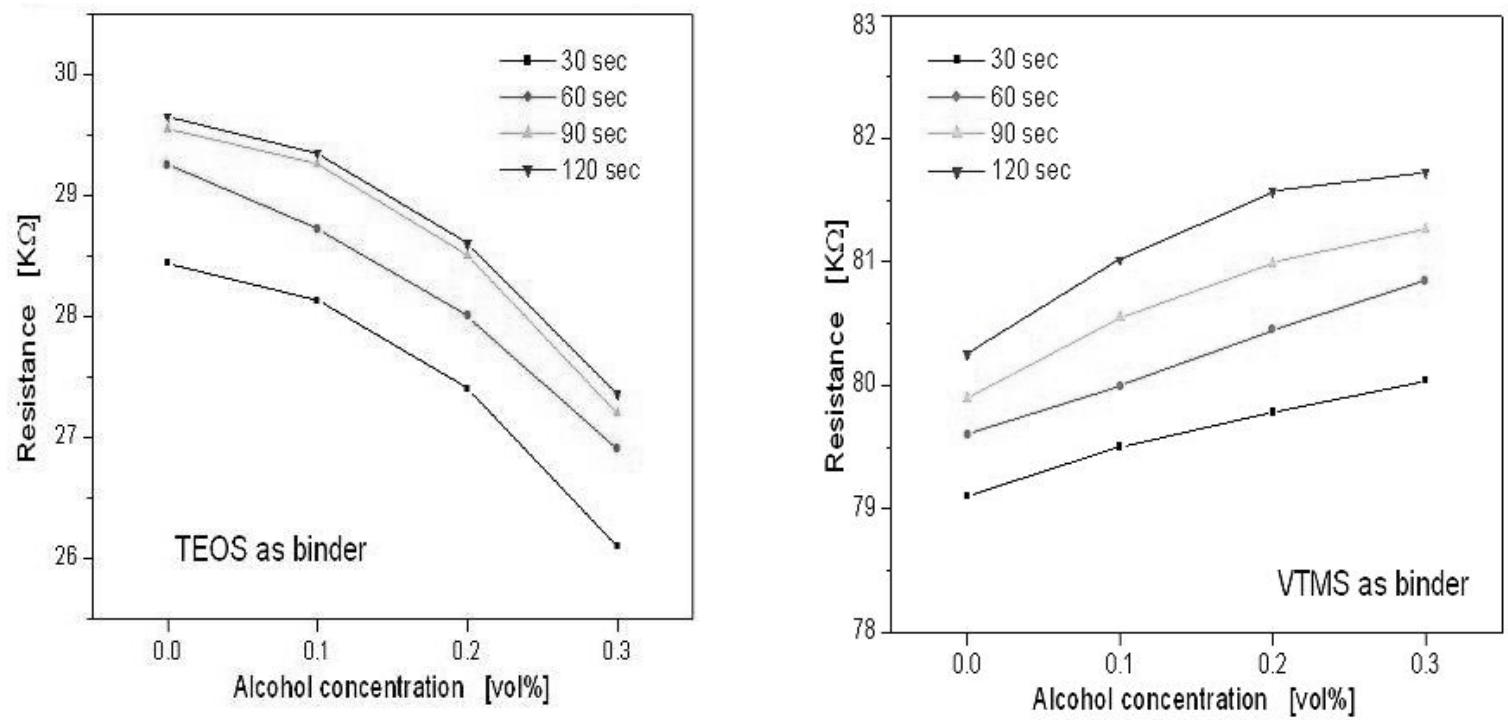

Fig. 3 The variation in resistance on the alcohol concentration as a function of the exposure time in the sensors using TEOS and VTMS as binder

\section{References}

[1] J. Kong, et. al., Science 287 (2000) p.622 625

[2] O. K. Varghese, et. al., Sens. Actuators B 81 (2001) p.32 41

[3] Y. Zhou, L. Hu and G. Gruner, Appl. Phys. Lett. 88 (2006) p.123109

[4] P. Qi, et al, Nano Lett. 3 (2003) p. 347-351 